\title{
Biotechnological aspects of improving the quality of young brandy distillates
}

\author{
Olga Chursina*, Viktor Zagorouiko, Ludmila Legasheva, Alina Martynovskaya, and Elena \\ Udod \\ Federal State Budget Scientific Institution All-Russian National Research Institute of Viticulture and \\ Winemaking Magarach of the RAS, 31 Kirova Str., 298600 Yalta, Republic of Crimea, Russia
}

\begin{abstract}
In connection with the increasing demand in high-quality raw materials for brandy production, the research intended to solve the problems of industry associated with using of low-acid or low-sugar grapes is relevant. A promising direction for improving the quality of brandy outputs is the use of yeasts with desired properties and yeast metabolism products. The aim of the research was to study the effect of biotechnological agents on physicochemical composition and quality of base wines and young brandy distillates. The experimental Kl.marxianusbased enzyme preparation with high endopolygalacturonase activity, races of yeast S.cerevisiae pure culture and L.thermotolerans strain from the Magarach collection of microorganisms of winemaking were used as biotechnological agents. Analytical studies were carried out using conventional methods, gas and liquid chromatography. The research results showed that using of yeast endopolygalacturonase based on Kl.marxianus helps to increase the yield of must (by $6 \%$ ). The L.thermotolerans strain is effective for increasing the titratable acidity of base wines (by 1.3-2.1 times). For grapes with low sugar content, it is advisable to use S.cerevisiae yeast races with a high ester-producing capacity.
\end{abstract}

\section{Introduction}

Winemaking technology is based on the yeast vital activity as a causative agent of alcoholic fermentation, resulting in biochemical transformations of grape carbohydrates into the key product - ethyl alcohol, and fermentation by-products - higher alcohols, esters, acids, etc. [1]. The most valuable production properties are attributed to the yeasts of Saccharomyces Meyen genus (1838), to which the majority of yeasts important in the fermentation industry belongs. The difference in biochemical characteristics of yeast races and their properties (alcohol and sulfite tolerance, cold resistance, etc.) under the influence of various environmental factors is determined by a wide range of synthesized volatile components and other products that create organoleptic properties of wine production.

Using of non-conditional grapes or non-traditional, including table, local or hybrid varieties in brandy production, leads to producing with poor or uncharacteristic profile of volatile components [2]. Control in aromatic substance composition of base wines and

\footnotetext{
* Corresponding author: olal45@mail.ru
} 
brandy distillates using the selected yeast races is one of the promising biotechnological methods to improve their quality.

At the same time, growing interest in recent years was attracted by non-Saccharomyces yeast genus, also possessing a number of useful properties for solving new problems of modern winemaking, one of which is the control of acidity in wine production by biological agents, without using of chemical reagents. The yeasts of Lachancea genus (Kurtzman, 2003), capable to synthesize lactic acid during must carbohydrate fermentation are increasingly used to raise the content of organic acids in wine in a number of winemaking regions with hot climate. In this case, the wine $\mathrm{pH}$ value can be shifted by 0.5 units or more [3], allowing to give fresh and balanced flavor, increase reliability and stability of wines [4, 5]. Along with acidity increase, yeasts of Lachancea genus have a number of other characteristics that increase wine quality $[6,7]$. Such a close attention of researchers allowed to include them in the list of 20 most frequently mentioned nutrient yeasts [8]. However, the industrial application of both these and most other non-Saccharomyces yeasts is hampered by low or moderate capacity to alcoholic fermentation, and the mechanisms of mixed fermentation, which can have not only synergic or additive effect on analytical profile of wine, but also a specific one, based on metabolic interaction of strains, are still in the process of research [9].

The high content of organic acids in base wines for brandy production contributes not only to protection from microbial spoilage and negative effect of oxidative processes, but also to intensification of ester formation during distillation, which is important for creating a brandy bouquet [10]. The biological method of increasing the acidity of base wines using L.thermotolerans yeasts is free from disadvantages of artificial acidification with organic acids, and opens up new possibilities for improving the quality of brandy products. However, there is no information on using L.thermotolerans in brandy production, which requires a comprehensive study.

Like many fruits, grape berries, depending on varietal characteristics and environmental growing conditions, can accumulate a significant level of polysaccharides, reducing the volume and clarification of the must and enriching it with undesirable components [11]. Using of industrial enzyme preparations of pectolytic action (Pectofoetidin, Pectinex, etc.) in brandy production is limited due to side effect and significant impact on the composition of volatile substances. Most of them have pectinesterase activity of varying degree, which leads to an increase in the content of strictly regulated methanol in the finished product. Extracellular yeast enzymes - endopolygalacturonases, produced by different yeast types, can work as their alternative. Their distinctive feature is that the content of proteins with endopolygalacturonase activity can reach $80-90 \%$ of the total cell proteins, and the number of forms of synthesized hydrolytic enzymes in yeast cultures is much less than in fungal cultures and bacteria.

Some yeast strains of Kluyveromyces genus (Filippov, Yarrow 1972) are highperformance producers of extracellular endopolygalacturonase [12]. Due to the capacity to ferment not only glucose, but also lactose, yeast abundance of Kluyveromyces genus in industrial conditions is very high [13]. However, despite obvious advantages of using, yeast pectolytic enzymes in brandy production have been studied insufficiently yet. In this regard, it seems promising to study the effect of endopolygalacturonase, produced by Kl.marxianus, on the composition and quality of brandy products.

Thus, the development of biotechnological approaches to solving acute problems of brandy production based on a more complete using of microorganism potential for targeted regulation of chemical and aroma-producing composition of brandy products is a relevant and promising research direction.

The aim of the research was to study the effect of biotechnological agents on physicochemical composition and quality of base wines and young brandy distillates. 


\section{Materials and Methods}

The research materials we used are must, base wines and young brandy distillates from grape varieties of different origin (introduced, local, interspecific) of 2015-2020 crop years, obtained in the conditions of micro-winemaking using the following biotechnological agents:

- experimental enzyme preparation (EP) obtained on the basis of culture fluid of III-360 strain (VKM U-848 strain), Kl.marxianus species, with endopolygalacturonase activity of 1422.22 units, introduced to freshly obtained not clarified must or pulp (dosage $-5 \mathrm{ml} / \mathrm{dm}^{3}$; exposure time $-1 \mathrm{~h}$; temperature $-18-20^{\circ} \mathrm{C}$ ). Then must was clarified with/without introduction of technological agents (enogelatin, dosage $-50 \mathrm{mg} / \mathrm{dm}^{3}$, and/or bentonite in a dosage $-0.5-1 \mathrm{~g} / \mathrm{dm}^{3}$, temperature $10-12^{\circ} \mathrm{C}$ ), racking and fermentation (race $47-\mathrm{K}$ );

- 12 races of pure yeast culture from the Magarach collection of microorganisms of winemaking (CMW Magarach) [14], S.cerevisiae species (47-K, Kokur 3, Artemovskaya 7, Magarach 17-35, Jerez 20S/96, Sevastopolskaya 23, Leningradskaya, Magarach 125, Feodosia I-19, Rkatsiteli 6, Sudak VI-5, Novotsimlyanskaya 3);

- L.thermotolerans strain, individually and in combination with S.cerevisiae (47-K, Artemovskaya 7, Sevastopolskaya 23, Jerez 20S/96).

Base wine distillation was carried out on a bench-scale plant using method of double distillation according to the Charente technology. A total of 18 grape varieties were studied, 226 samples of base wines and 243 samples of young brandy distillates were produced.

The chemical composition analysis of samples was carried out using conventional methods, Agilent Technology 6890 gas chromatograph with mass-spectrometric detector (HP-innowax quartz capillary column with helium gas carrier), and high-performance liquid chromatograph (Shimadzu LC20 AD Prominence, Japan). All chemical analyzes were performed in triplicate.

The organoleptic assessment of base wines and distillates was carried out involving the tasting panel of the FSBSI Institute Magarach of the RAS. The study results were systematized, processed by the methods of mathematical statistics, using correlation and regression analyzes and computer technology software.

\section{Results and Discussion}

The study results of yeast endopolygalacturonase during pulp processing showed an increase in the volume of must on average by $6 \%$ compared to the control (untreated pulp). Introduction of experimental EP into the must before its clarification using technological agents contributed to a more significant reduction in the content of suspended solids than when using only technological agents. This is based on a decrease in must viscosity under the effect of endopolygalacturonase and the content of polysaccharides (up to $20 \%$ ). The best results were obtained in the combined treatment with experimental EP, enogelatin and bentonite, decreasing the content of suspended solids in must by $43-48 \%$ (Fig. 1). Besides, in all variants with EP used, a lower volume of sedimentation and its more dense structure were observed in comparison with similar treatments without EP, contributing to an increase in the yield of the clarified must fraction (by 4-9\%).

Chemical analysis of further obtained base wines and young brandy distillates did not reveal a significant difference in the control and experimental samples, either in basic physicochemical parameters or in the composition of volatile components. It was found that the studied EP did not have a significant effect on the formation of methanol, the level of which in experimental samples did not exceed the level of the control.

Thus, the studies showed positive effect of experimental EP on the clarification processes and quality of brandy base wines and distillates, contributing to an increase in the 
yield of must, a decrease in the content of suspended solids and sediment volume, determining the efficiency and expediency of using yeast endopolygalacturonase in brandy production.

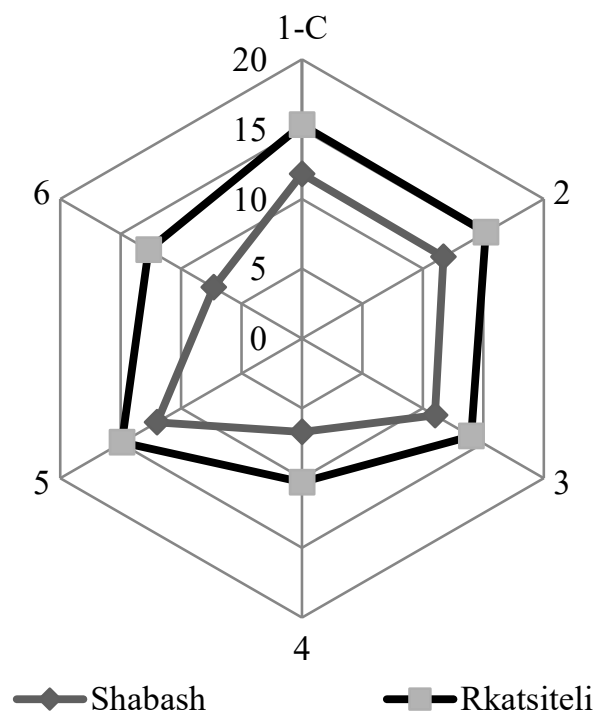

Fig. 1. The effect of must processing prepared from 'Shabash' and 'Rkatsiteli' grape varieties on the mass concentration of suspended solids, g/l: variants: 1-C - control (untreated); 2 - EP; 3 $\mathrm{EP}+$ bentonite; 4 - EP+enogelatin+bentonite; 5 - bentonite; 6 - enogelatin+bentonite

A shortage in the level of organic acids in berries, due to grape varietal characteristics, as well as arid climate in zones of traditional grape growing, associated with global warming, negatively affects the quality of brandy products. To regulate the acidity, L.thermotolerans was added to must during fermentation individually and together with S.cerevisiae. The base wine study results showed an increase in the mass concentration of titratable acids, based on the formation of lactic acid, in all experimental samples. The most significant increase in parameter values was observed during must fermentation of L.thermotolerans individually, by an average of 2.1 and 29.4 times, respectively, compared to the control (S.cerevisiae). In this case, the $\mathrm{pH}$ decrease reached 0.3 .

In base wines obtained in co-inoculating of L.thermotolerans and S.cerevisiae, changes in chemical composition were less significant: the mass concentration of titratable acids increased by an average of 1.3 times, and lactic acid - by an average of 4.1 times (Fig. 2). In this case, the $\mathrm{pH}$ value in individual experimental samples decreased by 0.1 . 


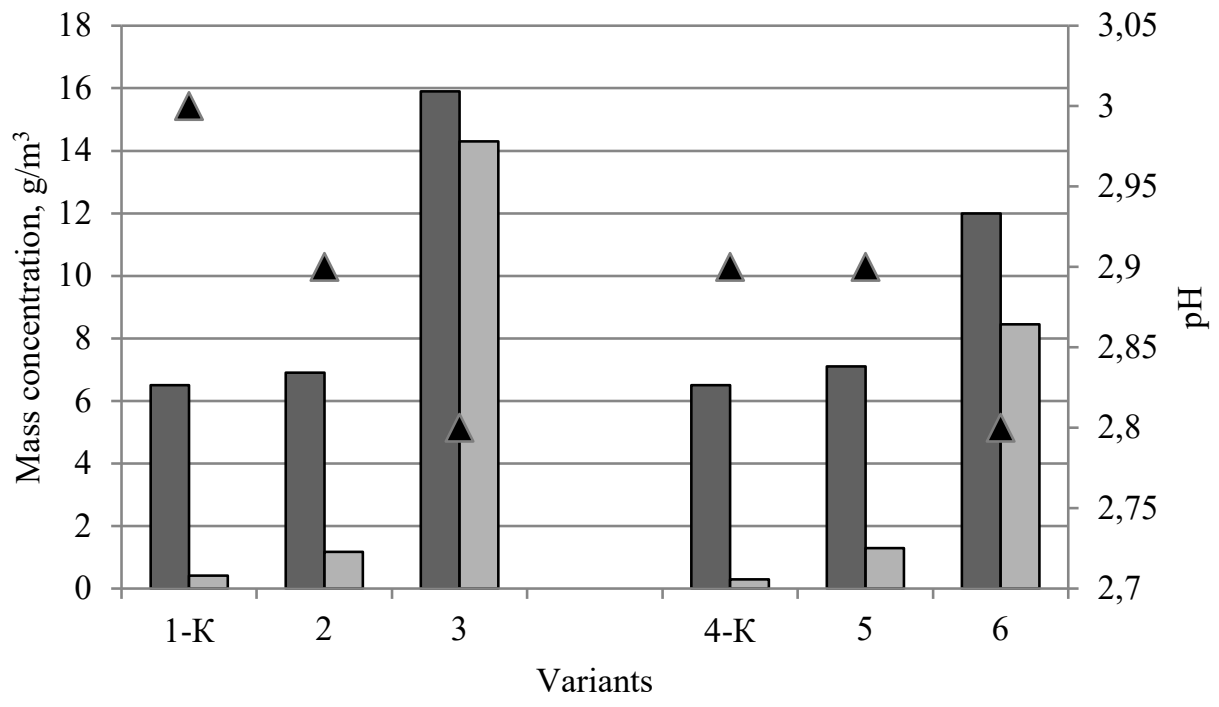

$\square$ titratable acids $\quad \square$ lactic acid $\quad \mathbf{\Delta} \mathrm{pH}$

Fig. 2. The effect of L.thermotolerans and S.cerevisiae strains on $\mathrm{pH}$, mass concentration of titratable acids and lactic acid of base wines: $1-\mathrm{K}-$ control $(47-K) ; 2-$ L.thermotolerans $+47-K ; 3,6-$ L.thermotolerans; 4-K - control (Magarach 17-35); 5 - L.thermotolerans + Magarach 17-35.

Chemical analysis of young brandy distillates obtained in further distillation of experimental base wines in comparison with the control (S.cerevisiae) showed an increase in the content of middle-chain esters due to the formation of an alcoholic ester of lactic acid. Its concentration also depended on the method of strain inoculation during must fermentation. During co-inoculation of L.thermotolerans and S.cerevisiae, the content of ethyl lactate in young brandy distillate increased by an average of 5.8 times, during individual inoculation - by 6.6 times, amounting to $3.1-5.5 \mathrm{mg} / \mathrm{dm}^{3}$ in the control. In experimental samples the content of ethyl acetate also increased by an average of 1.5 times and 2.3 times, respectively, amounting to $78.0 \mathrm{mg} / \mathrm{dm}^{3}$ in the control. The positive effect of using L.thermotolerans for brandy production may also consist in the revealed tendency of methanol decreasing in experimental samples - by an average of 1.1 times in co-inoculation with S.cerevisiae and 1.8 times - in individual inoculation.

Experimental base wines and young brandy distillates obtained in co-inoculation of L.thermotolerans and S.cerevisiae received the highest organoleptic assessment as were characterized by a more complex aroma with varietal notes and fresh flavor. The L.thermotolerans individual inoculation showed herbaceous tones and coarse sour flavor in base wines, and a bouquet simplification in distillates, reducing their assessment.

On the strength of the data obtained, it can be confirmed that using of L.thermotolerans together with S.cerevisiae in brandy production makes it possible to effectively regulate the titratable and active acidity of base wines, contributing to preservation of varietal grape aroma, an increase in the content of medium-chain esters and a decrease in the content of methanol, as well as raising of organoleptic characteristics of brandy products.

As far as is known, the main disadvantage in the composition of Russian brandy bouquet is the dominance of quality-reducing unpleasant fusel tones with intensity of 1.8 times higher than the level of fruit and flower descriptors. This is partly due to cropping and processing of technically unripe grapes, which leads to a low accumulation of aromatic 
substances and a high content of superior alcohols with coarse aroma in the finished product. More favorable conditions for yeast growth (glucose, nitrogenous substances, etc.) are created with an increase in the sugar content in grapes, which, along with an increase in reserve of aromatic substances in a berry, leads to an increase in the content of esters formed during alcoholic fermentation. So, according to our data, an increase in the mass concentration of sugars in grapes from $140 \mathrm{~g} / \mathrm{dm}^{3}$ to $170-190 \mathrm{~g} / \mathrm{dm}^{3}$ contributes to greater increase in the content of esters (up to $40 \%$ ) in base wines, first of all, due to the formation of ethyl acetate, ethyl lactate and diethyl succinate, as well as essential components of enanthic ester (ethyl caprylate, ethyl caprynate, etc.).

Chromatographic analysis of aromatic complex of homogeneous base wines obtained using 12 yeast races from CMW Magarach showed that the content of medium-chain esters can vary in a wide range (1.7-3.8 times) (Fig. 3). It allows regulating the composition of aromatic substances in base wines, using races in dependence with their synthesizing capacity. The studies show that the most promising for brandy production, especially when processing interspecific grape varieties, are the yeast races of S.oviformis Osterwalder species, 1924 (according to the classification of V.I.Kudryavtsev) or S.bayanus Saccardo (according to the Lodder classification): Jerez 20S/96, Magarach 17-35, Sevastopolskaya 23 , which perform an increased capacity to form esters. In addition, it was revealed that Jerez 20S/96 race is also distinguished by a moderate level of higher alcohol accumulation. The races Novotsimlyanskaya 3 and Artemovskaya 7 were marked by the lowest synthesis level of higher alcohols in comparison with other races. Their application allows harmonizing the aroma-producing composition of brandy products, the quality of which is associated with an increase in the ratio of medium-chain esters to higher alcohols (by 1.22.0 times).

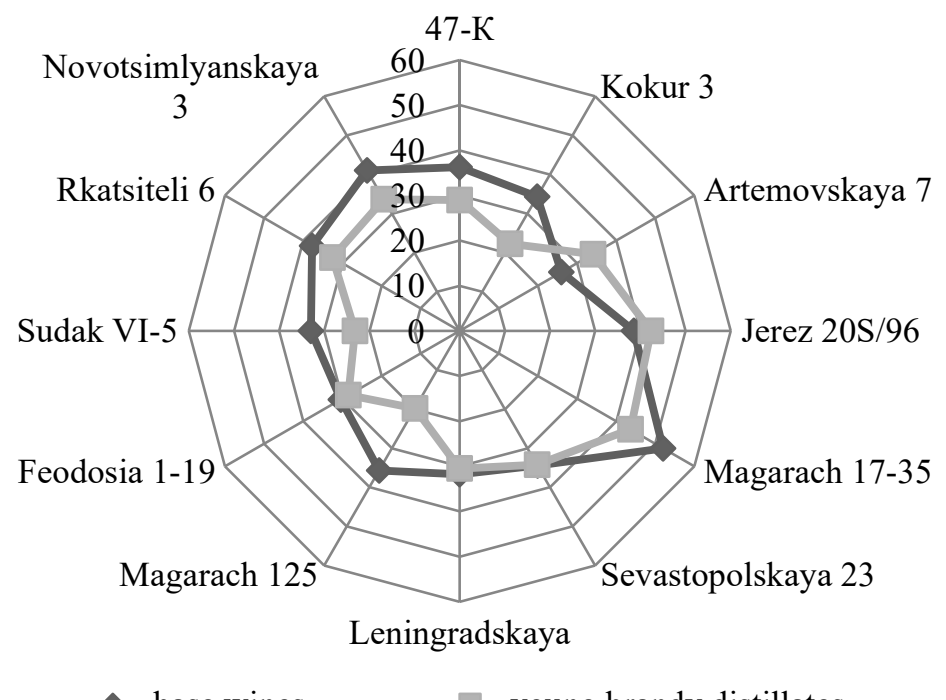

$\sim$ base wines $\quad-$ young brandy distillates

Fig. 3. The content of medium-chain esters in homogeneous base wines and young brandy distillates depending on the yeast race, $\mathrm{mg} / \mathrm{dm}^{3}$ (average values for base wines prepared from different grape varieties) 


\section{Conclusion}

The research results show the potential of biotechnological direction in brandy production using yeast strains with desired properties and their metabolic products with enzymatic activity to regulate the physicochemical and aroma-producing composition of brandy products:

- reducing the content of polysaccharides in must and pulp by introducing an experimental enzyme preparation with high endopolygalacturonase activity, obtained on the basis of Kl.marxianus species strain. Testing of experimental enzyme preparation showed its high efficiency, contributing to an increase in the yield of must (on average by $6 \%$ ), the degree of its clarification and the quality of brandy products;

- increasing the titratable acidity of base wines by using L.thermotolerans during must fermentation (by 1.3-2.1 times depending on the method of inoculation), contributing to preservation of varietal aroma of grapes, an increase in the content of medium-chain esters and a decrease in the concentration of methanol, as well as raising of organoleptic characteristics of brandy products;

- enrichment of the aroma-producing composition of base wines and young brandy distillates by using S.cerevisiae yeast races with an increased ester-producing capacity and a reduced synthesis of higher alcohols. It is established that the most promising, ensuring balanced aroma-producing composition of brandy products and improving its quality for brandy production are: Jerez 20S/96, Magarach 17-35 and Sevastopolskaya 23 yeast races.

The research results are especially relevant for development of raw material base in brandy production and enhancement of its varietal structure, in particular, based on local and introduced (European) varieties characterized by low titratable acidity, critical in conditions of global warming, as well as on cold-resistant interspecific varieties or grapes, not reached technical ripeness, with insufficient supply of aromatic substances. Application of recent developments will increase the efficiency of using grape biological potential, quality and competitiveness of brandy products, reduce costs and solve the problem of import substitution in brandy production.

\section{References}

1. J. Moreno, R. Peinado, Enological chemistry, 442 (London: Academic Press, 2012)

2. O. Chursina, V. Zagorouiko, L. Legasheva, A. Martynovskaya, M. Prostak, E3S Web of Conferences, 175, 08007 (2020)

3. A. Morata, I. Loira, W. Tesfaye, M.A. Bañuelos, C. González, J.A.S. Lepe, Fermentation, 4, 53, 12 (2018)

4. B.S. Chidi, F.F. Bauer, D. Rossouw, S. Afr. J. Enol. Vitic., 39 (2), 315-329 (2018)

5. A. Robles, M. Fabjanowicz, T. Chmiel, J. Płotka-Wasylka, TrAC Trends in Analytical Chemistry, 120, 115630 (2019)

6. A. Benito, F. Calderón, F. Palomero, S. Benito, Molecules, 20, 9510-9523 (2015)

7. M.L. Ponsone, M.C. Nally, M.L. Chiotta, M. Combina, J. Köhl, S.N. Chulze, Biol. Control, 103, 78-85 (2016)

8. S. Benito, Applied Microbiology and Biotechnology, 102, 6775-6790 (2018)

9. M. Ciani, F. Comitini, Current Opinion in Food Science, 1, 1-6 (2015)

10. S.M.G. Saerence, F.R. Delvaux, K.J. Verstrepen, J.M. Thevelein, Microb. Biotechnol., 3 (2), 165-177 (2010)

11. A.A. Watrelot, D.L. Schulz, J.A. Kennedy, Food Hydrocolloids, 63, 571-579 (2017) 
12. E.S. Naumova, A.Z. Sadykova, Y.V. Michailova, G.I. Naumov, Microbiology, 86 (3), 363-369 (2017)

13. S. Rollero, A.J.J. Zietsman, F. Buffetto, J. Schückel, A. Ortiz-Julien, B. Divol, J. Agric. Food Chem., 66 (44), 11739-11747 (2018)

14. T.N. Tanaschuk, S.A. Kishkovskaya, E.V. Ivanova, T.K. Skorikova, Collection of microorganisms of winemaking. Catalogue of cultures, 174 (Yalta: FSBSI Magarach, 2017) 